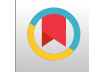

\title{
Knowledge, Attitude, and Practice Toward COVID-19 Among Healthcare Workers in Shiraz, Iran
}

\author{
Farnaz Kamali Haghighi (iD ${ }^{1}$, Pariya Kouhi (iD ${ }^{1,}{ }^{*}$, Mitra Amini (iD ${ }^{2}$, Vahid Mohammadkarimi (iD) ${ }^{1}$, Minoo \\ Sepehrpoor (iD ${ }^{3}$, Seyed Ali Hosseini (iD ${ }^{3}$, Mahsa Moosavi (iD) ${ }^{2}$ and Shahrokh Sadeghi Boogar (iD) 1 \\ ${ }^{1}$ Department of Internal Medicine, School of Medicine, Shiraz University of Medical Sciences, Shiraz, Iran \\ ${ }^{2}$ Clinical Education Research Centre, Shiraz University of Medical Sciences, Shiraz, Iran \\ ${ }^{3}$ Medical Intern, Student Research Committee, Shiraz University of Medical Sciences, Shiraz, Iran \\ "Corresponding author: Department of Internal Medicine, School of Medicine, Shiraz University of Medical Sciences, Zand St, P.O. Box: 71348-14336, Shiraz, Iran. Tel: \\ +98-9338919922, Email: kouhip@sums.ac.ir \\ Received 2020 August 26; Revised 2020 October 07; Accepted 2020 October 13.
}

\begin{abstract}
Background: The coronavirus disease 2019 (COVID-19) pandemic is a critical world concern, with healthcare workers (HCWs) at a high risk of infection. Thus, a sufficient level of knowledge, attitude, and practice (KAP) must be ensured.

Objectives: We aimed to investigate the KAP toward COVID-19 among HCWs in Shiraz, Iran.

Methods: A cross-sectional study was performed on a large sample of HCWs dealing with the COVID-19 pandemic in multiple hospitals affiliated to the Shiraz University of Medical Sciences. The survey featured four sections: demographic data, knowledge, attitude, and practice. An expert panel validated the questionnaire, and its reliability was ensured through a pilot study on 20 HCWs (Cronbach's alpha $=0.9$ ).

Results: We found that $72.8 \%$ of HCWs had sufficient knowledge, $66.65 \%$ indicated correct attitudes, and $67.72 \%$ described good practices. However, 24.23\% thought adequate protective equipment or facilities are unavailable. The knowledge scores were lowest on prevention and diagnosis, though symptoms were well-recognized. The male gender was correlated with more correct attitudes. Among nurses, 2 - 5 years of work experience was associated with better practice, with a master's degree being related to more positive attitudes.

Conclusions: The KAP of HCWs toward COVID-19 are acceptable in Shiraz, but further education and training is beneficial, especially on disinfectants and prevention; reassurance regarding protective equipment is also vital.
\end{abstract}

Keywords: Knowledge, Attitude, Practice, COVID-19, Healthcare Workers

\section{Background}

The coronavirus disease 2019 (COVID-19) is caused by a novel RNA virus of the coronavirus family, which is essentially a human and animal pathogen (1). The first case was reported on 30th December 2019 in China, ahead of transmission to almost all countries (2). The virus has given rise to a global pandemic (3).

Among the critical matters at hand are healthcare workers' (HCWs) infection and disease as well as the nosocomial spread of the illness. Healthcare workers are at the forefront of patient care. Every country allocates plenty of time and resources to the training of HCWs, who comprise valuable, irreplaceable members of society (4). In China, 3000 HCWs became infected and transmitted the disease to their respective families, making it essential to educate medical staff about COVID-19 and its prevention (3).

In pandemic conditions, every member of the global society is responsible for preventing the spread of the dis- ease, whether they are at home, at school, in the workplace, or anywhere else; the necessary precautions must always be taken. Transmission is usually more probable when the distance between persons is less than four feet and if they have direct contact with infected secretions such as sputum, blood, serum, and respiratory droplets (5). The World Health Organization (WHO) and Center for Disease Control and Prevention (CDC) recommend the following advice to protect HCWs from COVID-19 according to the mode of disease transmission: every patient suspected of an acute respiratory problem should be initially separated and must wear a face mask; frequent hand washing is necessary; and HCWs should use protective equipment for aerosolgenerating procedures during close patient contact $(6,7)$.

Infection with COVID-19 may cause a broad spectrum of symptoms ranging from a common cold to respiratory distress, dependence on mechanical ventilation, and death (8). 
Current diagnostic tests include the reverse transcription-polymerase chain reaction (RT-PCR) assay on respiratory secretions and the high-resolution CT scan. The sensitivity of RT-PCR is about $30-50 \%$ (9). Information about the disease is limited, but the incubation period seems to last for up to two weeks; comprehensive treatment is also not yet available (10).

Various mortality rates have been reported for COVID19, including $2.7 \%$ in China and an alarming $7.2 \%$ in Italy; this rate is about $2.9 \%$ in Iran, making its prevention an absolute necessity (11-13).

\section{Objectives}

The high rate of COVID-19 infections in Iran makes the education and training of HCWs on self-protection vital; health personnel must possess adequate knowledge and correct attitudes toward preventing disease occurrence. To date, no research has focused specifically on the knowledge, attitude, and practice (KAP) of public hospital HCWs toward COVID-19 in a multi-center sample in Iran. Hence, the present study aimed to investigate this topic among public HCWs in hospitals of Shiraz, Iran. This is vital given the pandemic state and the urgency of evaluation, training, and education. It is anticipated that by identifying deficits in education and prevention, those who are on the front lines of patient care and treatment can be assisted and protected, averting any impairments to patient care.

\section{Methods}

\subsection{Data Collection}

We conducted this cross-sectional study by a selfadministered questionnaire in multispecialty hospitals in Shiraz, Iran, from the 25th February 2020 to the 15th March 2020.

All of the physicians, nurses, medical students, and residents who worked in the mentioned hospitals were included in this study, and finally, 495 HCWs agreed to fill out our questionnaire.

A standard self-administered questionnaire was distributed among HCWs by two authors and explained to them if they had any problems completing the survey. An online version of the questionnaire was also made available. Ultimately, the responses of $495 \mathrm{HCWs}$ were collected.

\subsection{Questionnaire Design}

We reviewed the WHO and CDC guidelines and literature about COVID-19, then designed a self-administered questionnaire composed of four parts: (I) demographic information, (II) knowledge, and (III) attitude toward COVID19 , and (IV) practices of the HCWs. Validation took place in two steps. First, we revised the questions with a collaborative panel of professionals from the internal medicine and epidemiology departments of Shiraz University of Medical Sciences. Then, a pilot study was conducted with a small sample of 20 HCWs. Accordingly, the revised questionnaire proved reliable, with a Cronbach's alpha value of 0.9.

We designed 21 questions on the nature, source of transmission, symptoms, prevention, diagnosis, treatment, and prognosis of COVID-19 to facilitate a comprehensive evaluation of the knowledge of HCWs.

The attitudes of HCWs were assessed through 13 items, each asking how the respondent felt on a five-point Likert scale: (1) strongly agree, (2) agree, (3) undecided, (4) disagree, and (5) strongly disagree.

Our study assessed the practices of HCWs through nine questions with three options: yes, no, or proper equipment is unavailable.

\subsection{Data Analysis}

This study assessed the KAP of HCWs through a specific range of questions. The knowledge score ranged from 0 to 21; scores below 15 were considered to indicate a poor level of knowledge, while those greater than or equal to 15 signified sufficient knowledge.

In regard to attitude, the more frequent answer of 1 2 was considered to reveal a positive attitude, whereas a negative attitude was indicated by more frequent scores of three and above.

Our study assessed the practice of healthcare workers by nine questions with three choices: yes, no, proper equipment was not available (incorrect answer: 1, correct answer: 2). Total scores greater than or equal to seven for each questionnaire were regarded as indicators of good practice, whereas those below this cut-off signified inappropriate practice. The availability of protective equipment was also assessed.

The data were analyzed using the one-way analysis of variance and independent t-test functions of SPSS software.

Ultimately, the correlation of gender, age, and occupation with KAP were evaluated among all the HCWs, with the correlation of degree and work experience with KAP being evaluated only among nurses as they fulfilled a more permanent role in the hospital system than medical trainees.

The Medical Ethics Committee of Shiraz University of Medical Sciences approved this study (code number: IR.SUMS.med.rec.1399.027). We explained the aim of this study to participants, and they responded to the questionnaire consciously. 


\section{Results}

The study included 495 public HCWs who worked/trained at hospitals dependent on the Shiraz University of Medical Sciences; the demographic characteristics are summarized in Table 1.

\begin{tabular}{|c|c|}
\hline Features & No. (\%) \\
\hline \multicolumn{2}{|l|}{ Age group, y } \\
\hline$<25$ & $213(52.7)$ \\
\hline $26-36$ & $168(41.6)$ \\
\hline$\geq 37$ & $23(5.7)$ \\
\hline Total & $404(100)$ \\
\hline \multicolumn{2}{|l|}{ Gender } \\
\hline Male & $180(36.7)$ \\
\hline Female & $310(63.3)$ \\
\hline Total & $490(100)$ \\
\hline \multicolumn{2}{|c|}{ Nurses' work experience, y } \\
\hline$<2$ & $44(30.8)$ \\
\hline $2-5$ & $32(22.4)$ \\
\hline$>5$ & $67(46.8)$ \\
\hline Total & $143(100)$ \\
\hline \multicolumn{2}{|l|}{ Nurses' degree } \\
\hline Diploma & $6(4.2)$ \\
\hline Bachelor's & $131(91.6)$ \\
\hline Master's & $6(4.2)$ \\
\hline Total & $143(100)$ \\
\hline
\end{tabular}

The mean age was 26.84 years (range: 20 to 57 years). The majority of the HCWs were women ( $n=310$; 63.3\%). Nurses had the most work experience, with the bachelor's degree being the most common degree among them (n $=131 ; 91.65 \%$ ). Medical students (stagers) comprised the largest group of HCWs with a frequency of 31.85\% $(n=158)$, followed by nurses $(22.11 \%$; $112 \mathrm{n}=109)$. The minimum frequency was related to supervisors (0.84\%; $4 \mathrm{HCWs).}$

Overall, in regard to COVID-19, $72.8 \%$ of HCWs $(n=360)$ possessed adequate knowledge, $66.65 \%(n=329)$ had positive attitudes, and $67.72 \%(n=335)$ described good practice.

Notably, almost a quarter of participants $(24.23 \% ; n=$ 120 ) indicated a lack of the necessary equipment or facilities for the appropriate practices. Table 2 shows the complete questionnaire and the rate of correct responses to each question.

In terms of the COVID-19 knowledge subscales, the HCWs scored highest on knowledge pertaining to the symptoms of the disease (correct response rate: 95.6\%; n
$=473$ ). Conversely, the lowest knowledge scores were related to the category of diagnostic tests. Categorically, the remaining correct response rates were $68.5 \%(n=339)$ for transmission, 74.5\% $(n=369)$ for virology, 65.92\% $(n=326)$ for prevention, and $84.5 \%(\mathrm{n}=418)$ for treatment. Interestingly, only $14 \%(n=69)$ of HCWs knew that chlorhexidine is ineffective against the virus.

Regarding attitude toward COVID-19, 46\% of the HCWs $(\mathrm{n}=228)$ had a negative attitude about being exposed to infected patients. As the most common finding in this section, $94.7 \%(n=469)$ of them were worried about the transmission of infection to their loved ones. Only $2.8 \%$ of HCWs $(n=14)$ possessed a positive attitude about the availability of personal protective 128 equipment.

The independent $t$-test was employed to investigate the correlation between gender and age with KAP. The P-value was more than 0.05 in both men and women. There was not a significant correlation between gender and knowledge and practical skills. Although the mean practical skill score was more in women than men, only the positive attitudes related to COVID-19 possessed a significant correlation with the male gender $(\mathrm{P}<0.05)$. No other correlations were found between gender and either of the KAP variables. The results of one-way analysis of variance (ANOVA) revealed a lack of significant correlation between the nurses' degree and their KAP, though the Scheffe test revealed remarkably more good practice among nurses with work experience of 2 - 5 years than either extreme (Table 3 ).

The results of ANOVA (Table 4) elucidated a significant correlation between the occupation of the HCWs and their knowledge and attitudes ( $\mathrm{P}<0.05)$. The Scheffe test revealed that knowledge scores were significantly higher among medical students, residents, and general physicians relative to nurses and paramedics $(\mathrm{P}<0.0001)$.

Furthermore, differences in attitude were also significant $(\mathrm{P}<0.002)$, with medical residents having the most positive attitudes and general physicians achieving the minimum positive attitude in this regard.

\section{Discussion}

The COVID-19 pandemic is a global threat, with HCWs being present on the front lines of diagnosis, care, and treatment, making them at a higher risk of infection and disease. As valuable and much-needed members of society, HCWs must possess correct KAP about COVID-19 transmission, diagnosis, treatment, prevention, and prognosis.

This study revealed that $72.2 \%$ of HCWs $(n=360)$ had sufficient knowledge, 66.65\% ( $\mathrm{n}=329)$ had positive attitudes, and $67.72 \%(n=335)$ possessed adequate practical skills, but $15024.23 \%(n=120)$ believed the availability of protective equipment or facilities was not enough. 


\begin{tabular}{|c|c|c|c|}
\hline Variables & Number & Mean \pm SD & Test Statistic \\
\hline \multicolumn{4}{|c|}{ Work Experience, y } \\
\hline Practice & & & $\mathrm{F}=5.93, \mathrm{P}$ value $=0.003$ \\
\hline$<2$ & 41 & $14.65 \pm 4.93$ & \\
\hline $2-5$ & 26 & $15.38 \pm 5.26$ & \\
\hline$>5$ & 63 & $12.28 \pm 3.81$ & \\
\hline Knowledge, y & & & $\mathrm{F}=6.22, \mathrm{P}$ value $=0.003$ \\
\hline$<2$ & 34 & $72.67 \pm 3.95$ & \\
\hline $2-5$ & 27 & $71.70 \pm 3.41$ & \\
\hline$>5$ & 54 & $74.5 \pm 3.41$ & \\
\hline Attitude, $y$ & & & $\mathrm{~F}=4.13, \mathrm{P}$ value $=0.01$ \\
\hline$<2$ & 41 & $43.48 \pm 3.19$ & \\
\hline $2-5$ & 29 & $42.86 \pm 2.85$ & \\
\hline$>5$ & 65 & $44.72 \pm 3.25$ & \\
\hline \multicolumn{4}{|c|}{ Degree } \\
\hline Knowledge & & & $\mathrm{F}=0.24, \mathrm{P}$ value $=0.78$ \\
\hline Diploma & 6 & $12.66 \pm 3.2$ & \\
\hline Bachelor's & 118 & $13.76 \pm 4.75$ & \\
\hline Master's & 5 & $12.8 \pm 4.02$ & \\
\hline Attitude & & & $\mathrm{F}=2.57, \mathrm{P}$ value $=0.08$ \\
\hline Diploma & 3 & $28 \pm 7.21$ & \\
\hline Bachelor's & 109 & $32.59 \pm 3.37$ & \\
\hline Master's & 4 & $32.5 \pm 2.64$ & \\
\hline Practice & & & $\mathrm{F}=3.02, \mathrm{P}$ value $=0.04$ \\
\hline Diploma & 6 & $16.66 \pm 2.73$ & \\
\hline Bachelor's & 125 & $17.79 \pm 2.67$ & \\
\hline Master's & 5 & $20 \pm 2$ & \\
\hline
\end{tabular}

These results are comparable to some related studies. Regarding knowledge related to COVID-19, we found less sufficient knowledge compared to Chinese research and a study from Nigeria. Still, our study showed sufficient knowledge compared to studies done by Bhagavathula et al. and Olum et al. $(14,15)$.

Zhong et al. (16) studied 1357 HCWs from Henan province in April 2020 and showed 89\% sufficient knowledge among them. A Nigerian study assessed knowledge, attitude, and practice of 300 HCWs and revealed more than $90 \%$ correct answers to questions toward knowledge. (17) This difference between our study and others may be because of the fact that we assessed knowledge of COVID-19 in greater detail. Therefore, we found more defects in knowledge. Our result may be more close to reality. Ogolodom et al. (17) from Nigeria revealed better knowledge, but it may be due to some of their directional questions. Although we asked more questions about knowledge, we found better knowledge compared with the other two studies, and we believe this to be a positive sign.

Regarding attitude, our study is relatively similar to the other three pieces of research (14-16). However, the study done in Uganda showed a significantly less positive attitude, and the participants were concerned about the availability of personal protective equipment as were those in a study in Pakistan $(17,18)$.

Similar to our study, other studies found better knowledge in physicians; this is expectable, and we should train other healthcare workers with more intensity.

Compared with the Henan and Uganda study, we found less sufficient practice. HCWs who participated in our study thought there were significant deficiencies and re- 


\begin{tabular}{|c|c|c|c|}
\hline Variable & Number & mean $\pm S D$ & Test Statistic \\
\hline Practice & & & $\mathrm{F}=0.98, \mathrm{P}$ value $=0.41$ \\
\hline Paramedic & 10 & $13 \pm 3.85$ & \\
\hline Nurse & 112 & $13.64 \pm 4.59$ & \\
\hline Medical student & 218 & $14.4 \pm 4.19$ & \\
\hline Residents & 64 & $14.28 \pm 3.98$ & \\
\hline General physician & 58 & $13.67 \pm 3.42$ & \\
\hline Knowledge & & & $\mathrm{F}=18.17, \mathrm{P}$ value $<0.0001$ \\
\hline Paramedic & 5 & $71.2 \pm 2.28$ & \\
\hline Nurse & 103 & $73.37 \pm 3.63$ & \\
\hline Medical student & 216 & $76.01 \pm 3.53$ & \\
\hline Resident & 60 & $76.15 \pm 2.48$ & \\
\hline General physician & 56 & $77.18 \pm 2.67$ & \\
\hline Attitude & & & $\mathrm{F}=4.23, \mathrm{P}$ value $=0.002$ \\
\hline Paramedic & 10 & $43 \pm 2.26$ & \\
\hline Nurse & 116 & $44.09 \pm 3.37$ & \\
\hline Medical student & 218 & $44.04 \pm 3.06$ & \\
\hline Resident & 64 & $45.17 \pm 3.05$ & \\
\hline General physician & 58 & $18.96 \pm 3.41$ & \\
\hline
\end{tabular}

vealed practical skills less than others. In contrast, HCWs related to the Uganda study showed better practice despite a negative attitude about the availability of personal protective equipment.

We found an association between male gender and a more positive attitude and an association between more sufficient practical skills and nurses' work experience. The Henan study also showed better practical skills among nurses with higher work experience. We should be aware of this problem, HCWs with lower work experience may need more training programs.

Among the earliest studies conducted on HCWs in Iran in this field is that of Nemati et al. (19), who assessed the knowledge and anxiety of 85 nurses toward COVID-19. They found that only $56.5 \%$ of participants had sufficient knowledge, though our results were more satisfactory in this regard.

Recently, Maleki et al. (20) conducted a related study on 191 HCWs in a 185 single center in Kermanshah, Iran. In line with our results, these researchers found awareness about the method of transmission to be excellent, but the percentage of HCWs who failed to recognize the COVID-19 symptoms was $14 \%$ higher. Our results showed that the best knowledge of HCWs was about the symptoms of the disease, with nearly all respondents answering correctly in this regard. Conversely, the lowest level of knowledge was related to diagnostic tests. In the Nemati et al. study, it was shown that the lowest level of knowledge among nurses was about COVID-19 protection, advanced disease symptoms, and the incubation period.

Differences in outcomes between the studies may be due to the varied timings and the different composition of HCWs. It is important for health systems to recognize that intervention programs that merely boost knowledge levels do not suffice, and measures must be taken to promote correct practices in relation to infectious diseases.

The strong point of this study was that it included a high number of HCWs from two local centers with various professions, so the results are very useful for the local training program.

We also assessed knowledge of HCWs with more necessary detail. The results are useful in evaluating the needs, concerns, and possible lack of facilities; they can help in planning the required programs and in allocating the necessary protective equipment.

Nonetheless, this study had some limitations. For example, a high proportion of medical students, particularly those in their early years of training, were required to remain at home during the pandemic. We attempted to alleviate this problem through the use of online questionnaires, though the responses may not be as relevant as those obtained from medical students present in the 
healthcare centers.

Furthermore, factors that are predictive of KAP levels during outbreaks should be examined more thoroughly in future research.

In conclusion, there is an acceptable level of KAP regarding COVID-19 among HCWs in the city of Shiraz, Iran. However, further education is beneficial, with a particular need for training regarding disinfectant materials and prevention. Some of the HCWs believed the availability of protective equipment and facilities was inadequate, stressing the importance of reassurance in this regard because personal protective equipment will be available.

References

\section{Footnotes}

Authors' Contribution: Study concept and design: FKH, PK, and MA. Acquisition of data: PK, MS, and MM. Data analysis and interpretation: VMK, SAH, and SSB. Drafting of the manuscript: PK, FKH, MS, and MM. Critical revision of the manuscript for important intellectual content: SAH, SSB, MA, and VMK. Statistical analysis: MA. Study supervision: PK, and FKH. All authors approved the final version and accept accountability for the work.

Conflict of Interests: None declared.

Ethical Approval: The Medical Ethics Committee of Shiraz University of Medical Sciences approved this study [code: IR.SUMS.MED.REC.1399.027].

Funding/Support: This work was supported by the Shiraz University of Medical Sciences under grant no. 98-0101-21937.

\section{References}

1. Sahin AR, Erdogan A, Agaoglu PM, Dineri Y, Cakirci AY, Senel ME. 2019 novel coronavirus (COVID-19) outbreak: A review of the current literature. Eurasian J Med Oncol. 2020. doi: 10.14744/ejmo.2020.12220.

2. Chang TJ, Yang DM, Wang ML, Liang KH, Tsai PH, Chiou $\mathrm{SH}$, et al. Genomic analysis and comparative multiple sequences of SARS-CoV2. J Chin Med Assoc. 2020;83(6):537-43. doi: 10.1097/JCMA.0000000000000335. [PubMed: 32349035].

3. Adams JG, Walls RM. Supporting the health care workforce during the COVID-19 global epidemic. JAMA. 2020;323(15):1439-40. doi: 10.1001/jama.2020.3972. [PubMed: 32163102].

4. Shereen MA, Khan S, Kazmi A, Bashir N, Siddique R. COVID-19 infection: Origin, transmission, and characteristics of human coronaviruses. J Adv Res. 2020;24:91-8. doi: 10.1016/j.jare.2020.03.005. [PubMed: 32257431]. [PubMed Central: PMC7113610].

5. $\mathrm{Wu} \mathrm{Z}$, McGoogan JM. Characteristics of and important lessons from the coronavirus disease 2019 (COVID-19) outbreak in china: Summary of a report of 72314 cases from the Chinese center for disease control and prevention. JAMA. 2020;323(13):1239-42. doi: 10.1001/jama.2020.2648. [PubMed: 32091533].
6. CDC. Interim infection prevention and control recommendations for patients with suspected or confirmed coronavirus disease 2019(COVID-19) in healthcare settings. United States: Centers for Disease Control and Prevention; 2020.

7. Chughtai AA, Seale H, Islam MS, Owais M, Macintyre CR. Policies on the use of respiratory protection for hospital health workers to protect from coronavirus disease (COVID-19). Int J Nurs Stud. 2020;105:103567. doi: 10.1016/j.ijnurstu.2020.103567. [PubMed: 32203757]. [PubMed Central: PMC7174826].

8. Lauer SA, Grantz KH, Bi Q, Jones FK, Zheng Q, Meredith HR, et al. The in cubation period of coronavirus disease 2019 (COVID-19) from publicly reported confirmed cases: Estimation and application. Ann Intern Med. 2020;172(9):577-82. doi: 10.7326/M20-0504. [PubMed: 32150748]. [PubMed Central: PMC7081172].

9. Ai T, Yang Z, Hou H, Zhan C, Chen C, Lv W, et al. Correlation of chest ct and rt-PCR testing for coronavirus disease 2019 (COVID19) in China: A report of 1014 cases. Radiology. 2020;296(2):E32-40. doi: 10.1148/radiol.2020200642. [PubMed: 32101510]. [PubMed Central: PMC7233399].

10. Cascella M, Rajnik M, Cuomo A, Dulebohn SC, Di Napoli R. Features, evaluation and treatment coronavirus (COVID-19). Treasure Island (FL): StatPearls; 2020, [updated 2020 Oct 4].

11. Epidemiology Working Group for Ncip Epidemic Response. [The epidemiological characteristics of an outbreak of 2019 novel coronavirus diseases (COVID-19) in China]. Zhonghua Liu Xing Bing Xue Za Zhi. 2020;41(2):145-51. China. doi: 10.3760/cma.j.issn.02546450.2020.02.003. [PubMed: 32064853].

12. Abdi M. Coronavirus disease 2019 (COVID-19) outbreak in Iran: Actions and problems. Infect Control Hosp Epidemiol. 2020;41(6):754-5. doi: 10.1017 /ice.2020.86.

13. Onder G, Rezza G, Brusaferro S. Case-fatality rate and characteristics of patients dying in relation to COVID-19 in Italy.JAMA. 2020;323(18):17756. doi: 10.1001/jama.2020.4683. [PubMed: 32203977].

14. Olum R, Chekwech G, Wekha G, Nassozi DR, Bongomin F. Coronavirus disease-2019: Knowledge, attitude, and practices of health care workers at makerere university teaching hospitals, Uganda. Front Public Health. 2020;8:181. doi: 10.3389/fpubh.2020.00181. [PubMed 32426320]. [PubMed Central: PMC7204940].

15. Bhagavathula AS, Aldhaleei WA, Rahmani J, Mahabadi MA, Bandari DK Knowledge and perceptions of COVID-19 among health care workers: Cross-sectional study.JMIR Public Health Surveill.2020;6(2). e19160. doi: 10.2196/19160. [PubMed: 32320381]. [PubMed Central: PMC7193987].

16. Zhang M, Zhou M, Tang F, Wang Y, Nie H, Zhang L, et al. Knowledge, attitude, and practice regarding COVID-19 among healthcare workers in Henan, China. J Hosp Infect. 2020;105(2):183-7. doi: 10.1016/j.jhin.2020.04.012. [PubMed: 32278701]. [PubMed Central: PMC7194961].

17. Ogolodom MP, Mbaba A, Alazigha N, Erondu OF, Egbe NO, Golden I, et al. Knowledge, attitudes and fears of healthcare workers towards the corona virus disease (COVID-19) pandemic in south-south, Nigeria. Health Sci J. 2020. doi: 10.36648/1791-809x.s1.002.

18. Saqlain M, Munir MM, Rehman SU, Gulzar A, Naz S, Ahmed Z, et al Knowledge, attitude, practice and perceived barriers among healthcare workers regarding COVID-19: a cross-sectional survey from Pakistan.J Hosp Infect. 2020;105(3):419-23. doi: 10.1016/j.jhin.2020.05.007. [PubMed: 32437822]. [PubMed Central: PMC7211584].

19. Nemati M, Ebrahimi B, Nemati F. Assessment of Iranian nurses' knowledge and anxiety toward COVID-19 during the current outbreak in Iran. Arch Clin Infect Dis. 2020;15(COVID-19). e102848. doi: 10.5812/archcid.102848.

20. Maleki S, Najafi F, Farhadi K, Fakhri M, Hosseini F, Naderi M. Knowledge, attitude and behavior of health care workers in the prevention of COVID-19. researchsquare. 2020. doi: 10.21203/rs.3.rs-23113/v1. 
Table 2. Complete Questionnaire and Correct Response Rates

\begin{tabular}{|c|c|}
\hline Knowledge & Correct Response, \% \\
\hline \multicolumn{2}{|l|}{ Virology } \\
\hline Until now, the primary animal reservoir is not known exactly. & $54.5(n=270)$ agree/strongly agree \\
\hline \multicolumn{2}{|l|}{ Clinical manifestation } \\
\hline $\begin{array}{l}\text { In my country, this disease should be suspected if patients have a history of } \\
\text { travelling to China. }\end{array}$ & $93.1(n=461)$ disagree/strongly disagree \\
\hline The incubation period of this disease may be up to two weeks. & $94.5(n=468)$ agree/strongly agree \\
\hline The main symptoms of the disease are fever, cough, and shortness of breath. & $99.2(\mathrm{n}=491)$ agree/strongly agree \\
\hline \multicolumn{2}{|l|}{ Transmission } \\
\hline Currently, the main mode of disease transmission is human to human. & $80.4(n=398)$ agree/strongly agree \\
\hline The virus mostly transmits through respiratory droplets. & $83.9(n=415)$ agree/strongly agree \\
\hline Disease transmission by animal products is not known. & $54.7(n=280)$ agree/strongly agree \\
\hline You should not use meat and other animal products during this outbreak. & $72.7(n=360)$ disagree/strongly disagree \\
\hline The disease transmits mainly via the air. & $51.6(n=255)$ disagree/strongly disagree \\
\hline Disease transmission from asymptomatic individuals is possible. & $94.6(n=468)$ agree/strongly agree \\
\hline Pets may be infected, and transmission from pets is not known. & $39.3(n=194)$ agree/strongly agree \\
\hline \multicolumn{2}{|l|}{ Prevention } \\
\hline Currently, the main method of diagnosis is RT PCR. & $84.8(n=420)$ agree/strongly agree \\
\hline Currently, the main method of diagnosis is the antibody kit. & $45.6(n=226)$ disagree, strongly disagree \\
\hline Ethanol 70\% kills the virus. & $82.9(n=410)$ agree/strongly agree \\
\hline Chlorhexidine kills this virus. & $14(n=69)$ disagree/strongly disagree \\
\hline The most important way to prevent this disease is frequent handwashing. & $97(n=480)$ agree/strongly agree \\
\hline The most important way to prevent this disease is by wearing a mask. & $50.6(n=250)$ disagree/strongly disagree \\
\hline So far, no effective vaccine has been developed for the virus. & $86(n=426)$ agree/strongly agree \\
\hline \multicolumn{2}{|l|}{ Treatment } \\
\hline The exact treatment for the disease has not been determined yet. & $88.4(\mathrm{n}=438)$ agree/strongly agree \\
\hline \multirow[t]{2}{*}{ All patients with this disease need hospital admission. } & $79.7(n=394)$ disagree/strongly disagree \\
\hline & Positive Attitude, \% \\
\hline \multicolumn{2}{|l|}{ Attitude } \\
\hline I fear caring for COVID-19 patients. & $30.7(n=152)$ disagree/strongly disagree \\
\hline I fear working in a hospital where COVID-19 patient is admitted. & $41.5(n=205)$ disagree/strongly disagree \\
\hline My fear is relieved by protective equipment. & $73.8(n=365)$ agree/strongly agree \\
\hline Healthcare workers become infected at a high rate, even with enough protection & $32.9(n=163)$ disagree/strongly disagree \\
\hline I think the disease will finally come under control. & $64.5(\mathrm{n}=319)$ agree/strongly agree \\
\hline I think this disease cause serious economic damage. & $82.7(n=409)$ agree/strongly agree \\
\hline I believe that I may transmit this disease to my family. & $94.7(n=469)$ agree/strongly agree \\
\hline I must report it to my center if I become infected with this disease. & $80.3(n=397)$ agree/strongly agree \\
\hline Crowded places such as schools and mosques should be closed. & $95.6(n=473)$ agree/strongly agree \\
\hline Health education programs are effective for better control of the disease. & $98.2(n=486)$ agree/strongly agree \\
\hline $\begin{array}{l}\text { Wearing a surgical face mask and gloves is enough during the usual physical } \\
\text { examination. }\end{array}$ & $70.1(\mathrm{n}=347)$ agree/strongly agree \\
\hline $\begin{array}{l}\text { I should use an N95 face mask, face shield, gloves, and a gown when exposed to the } \\
\text { patient's secretions (e.g. CPR or suction of respiratory secretions). }\end{array}$ & $98.8(n=489)$ agree/strongly agree \\
\hline The necessary personal protective equipment is available. & $2.8(\mathrm{n}=13)$ agree/strongly agree \\
\hline I fear caring for COVID-19 patients. & $30.7(n=152)$ disagree/strongly disagree \\
\hline I fear working in a hospital where COVID-19 patient is admitted. & $41.5(n=205)$ disagree/strongly disagree \\
\hline My fear is relieved by protective equipment. & $73.8(n=365)$ agree/strongly agree \\
\hline Healthcare workers become infected at a high rate, even with enough protection & $32.9(n=163)$ disagree/strongly disagree \\
\hline I think the disease will finally come under control. & $64.5(n=319)$ agree/strongly agree \\
\hline I think this disease cause serious economic damage. & $82.7(n=409)$ agree/strongly agree \\
\hline
\end{tabular}


I believe that I may transmit this disease to my family.

I must report it to my center if I become infected with this disease.

Crowded places such as schools and mosques should be closed.

Health education programs are effective for better control of the disease.

Wearing a surgical face mask and gloves is enough during the usual physical examination.

I should use an N95 face mask, face shield, gloves, and a gown when exposed to the patient's secretions (e.g. CPR or suction of respiratory secretions).

The necessary personal protective equipment is available.

Practice

Do you use personal protective equipment when you visit a susceptible patient?

Do you wear a surgical face mask when doing the usual physical examination?

Do you wear an N95 face mask when doing aerosol-generating procedures?

Do you test the N95 mask before using it?

Do you isolate susceptible patients as soon as they arrive?

Do you wash your hands after physical examination or care of patients?

Do you recommend home isolation to anyone who contacted a patient infected with COVID-19?

If you have to care for more than one patient in the same place, do you apply a minimum distance of 4 feet? $94.7(n=469)$ agree/strongly agree

$80.3(\mathrm{n}=397)$ agree/strongly agree

95.6 (473) agree/strongly agree

$98.2(\mathrm{n}=486)$ agree/strongly agree

$70.1(n=347)$ agree/strongly agree

$98.8(n=489)$ agree/strongly agree

$2.8(\mathrm{n}=13)$ agree/strongly agree

Correct Response(Yes) Rate, \%

Lack of Facilities or Equipment, \%

$\begin{array}{ll}54.9(\mathrm{n}=272) & 43.9(\mathrm{n}=214) \\ 64.2(\mathrm{n}=318) & 27.7(\mathrm{n}=137) \\ 56.2(\mathrm{n}=278) & 40.1(\mathrm{n}=197) \\ 35(\mathrm{n}=173) & 36.3(\mathrm{n}=178) \\ 69(\mathrm{n}=342) & 26.7(\mathrm{n}=131) \\ 90.1(\mathrm{n}=46) & 4.7(\mathrm{n}=23) \\ 89.9(\mathrm{n}=445) & 6.7(\mathrm{n}=33) \\ 64.2(\mathrm{n}=318) & 26.3(\mathrm{n}=130)\end{array}$

